\title{
Effect of interfaces in Monte Carlo computer simulations of ferroelectric materials
}

\author{
D. Bolten, U. Böttger, ${ }^{\text {a) }}$ and R. Waser \\ Institut für Werkstoffe der Elektrotechnik, University of Aachen, D-52056 Aachen, Germany
}

(Received 5 May 2003; accepted 27 January 2004)

\begin{abstract}
In this article, Monte Carlo simulation methods were used to investigate the influence of interface layers between the ferroelectric core material and the electrodes on the hysteresis loop in ferroelectric thin films. The hysteresis loops were calculated using an existing Monte Carlo model. For certain interface configurations, the simulations resulted in asymmetric hysteresis loops, similar to imprinted loops, due to asymmetric nucleation kinetics. Although the results might not offer a new explanation for imprint in ferroelectric thin films, they provide insight into the often observed phenomenon of initially imprinted hysteresis loops of as-prepared thin-film samples. (C) 2004 American Institute of Physics. [DOI: 10.1063/1.1687455]
\end{abstract}

It was recently assumed that the discrepancies in electrical properties between thin films and bulk ceramics-for example, the thickness dependence of the coercive field, the decrease of the dielectric constant, or the switching properties of ferroelectric thin films-were due to the existence of a (possibly) nonferroelectric interface layer. ${ }^{1-4}$ Additionally, the imprint effect might be related to an interface between the bulk of the film and its electrodes. ${ }^{5,6}$ Several models have been proposed to account for the existence of such an interface layer, such as nonstoichiometric growth of the ferroelectric at the interface between the electrodes and the film due to interdiffusion or a stress-induced interface layer. ${ }^{7}$ Even though it has not been possible to provide direct verification of an interface layer, there has been ample indirect evidence for its existence. In this letter, the influence of interface layers on the shape of the hysteresis curves will be analyzed using Monte Carlo simulation methods.

The two-dimensional Monte Carlo simulation used to calculate the hysteresis loops was based on a model published by Potter et al. ${ }^{8}$ The model takes into account the dipole-dipole interaction, the gradient energy, and the energy of the dipoles in an external field. Details on the model and its implementation can be found in Ref. 9. To calculate a hysteresis loop, the simulation started with a completely random configuration of the simulation volume, usually of size $100 \times 100$, that was allowed to evolve for 400 Monte Carlo steps per spin (MCS/spin). After this initialization step, an electric field was applied along the $y$ direction of the simulation volume and changed stepwise to trace a complete hysteresis loop. At each field increment, the configuration was allowed to relax for $200 \mathrm{MCS} / \mathrm{spin}$, after which the configuration was saved to a file and the current polarization value was recorded. Free boundary conditions were employed.

To model the interface, different approaches were incorporated into the simulation. One interface model was the existence of a thin layer near the electrodes in which the value of the spontaneous polarization decreased slowly from the bulk of the material towards the surface. Another model

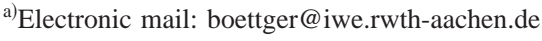

was the assumption of a thin interface layer near the electrodes in which the polarization is not allowed to switch. In real systems, this might be caused by the mechanical clamping effect of the substrate. The following discussion will focus on the latter model since the first had no visible effect on the shape of the hysteresis loop.

In this interface model, a thin layer at the top and bottom surface of the simulation volume (usually taken to be five lattice constants thick) was introduced, in which the polarization was assumed to be frozen in. The polarization orientation of these interfaces could either be chosen uniformly or randomly. The adoption of random interfaces (i.e., no correlation between neighboring lattice cells) resulted in no visible changes in the shape of the simulated hysteresis loops, as did the adoption of asymmetric interface configurations (e.g., a downwards polarized interface layer at the bottom electrode and an upwards polarized interface at the top electrode). In this case, however, there is a marked difference in how the nucleation and growth during the switching process proceeded. While, for a system with no interface layers, the nucleation and growth of oppositely oriented domains started from both electrodes, the nucleation and growth for a system with asymmetric interfaces is limited to one interface. In the case of switching the polarization from positive remanence to negative saturation, the nucleation of new domains started exclusively from the downwards polarized bottom interface, while for the reverse process (i.e., from negative remanence to positive saturation), the nucleation and growth started from the upwards polarized top interface. In both cases, no nucleation and growth occurred at the other side of the simulation volume. The resulting hysteresis curve (Fig. 1, no symbols) is symmetrical since the growth kinetics was identical for both processes.

A completely different pattern emerged when a symmetric interface was modeled; that is, both interfaces uniformly upwards polarized. The resulting hysteresis curve is also displayed in Fig. 1 (solid symbols). Here, the hysteresis curve is severely distorted and shifted along the electric field axis. Since both interfaces were uniformly polarized upwards the nucleation and growth of downwards polarized domains at the interfaces was severely impeded. Hence, the nucleation 


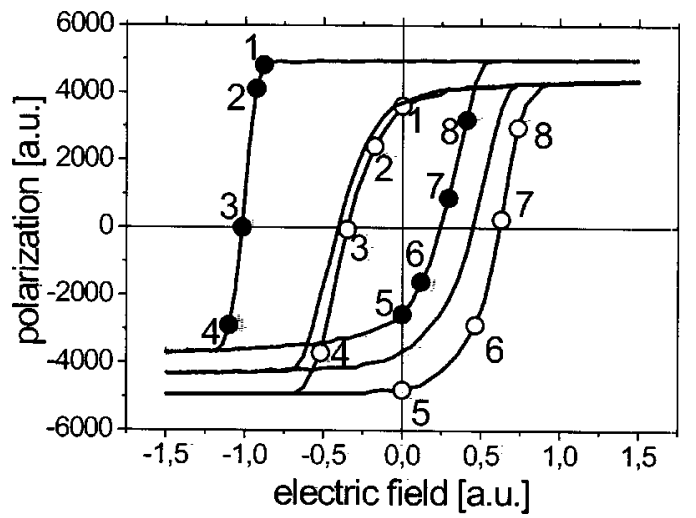

FIG. 1. Simulated hysteresis curves for different configurations of the top and bottom interfaces. Solid symbols: top and bottom interface upwards polarized. Open symbols: bottom interface downwards polarized. No symbols: top interface upwards polarized and bottom interface downwards polarized.

of downwards polarized domains proceeded by homogeneous nucleation and growth in the bulk of the simulation volume, as can be seen from Fig. 2, parts 1-4. The subfigure enumeration corresponds to the hysteresis states of Fig. 1. Since the energy barrier for homogeneous nucleation is
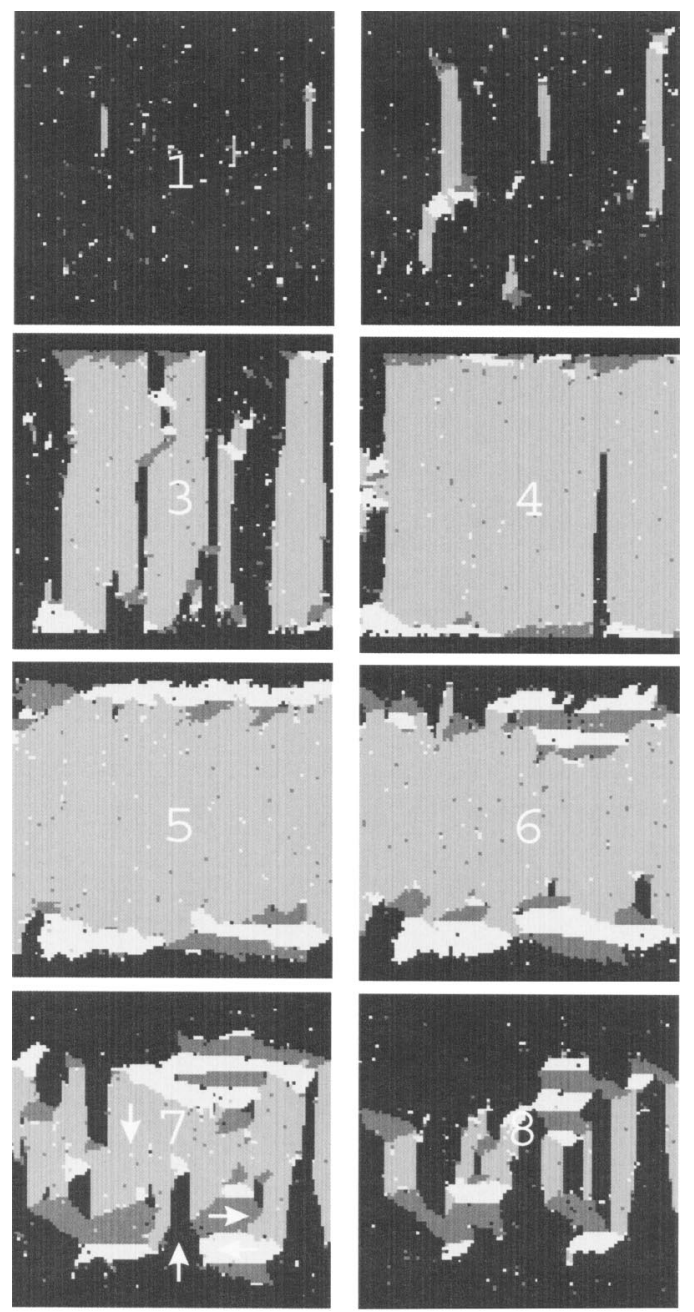

FIG. 2. Domain configurations during switching of a $100 \times 100$ lattice with frozen-in, upwards polarized top and bottom interfaces. The numbers correspond to the points in Fig. 1 (solid symbols). The polarization direction in the domains is represented by different shades of gray. The correspondence

is visualized in subfigure 7.
Downloaded 21 Dec 2006 to 134.94.122.39. Redistribution subject to AIP license or copyright, see http://apl.aip.org/apl/copyright.jsp
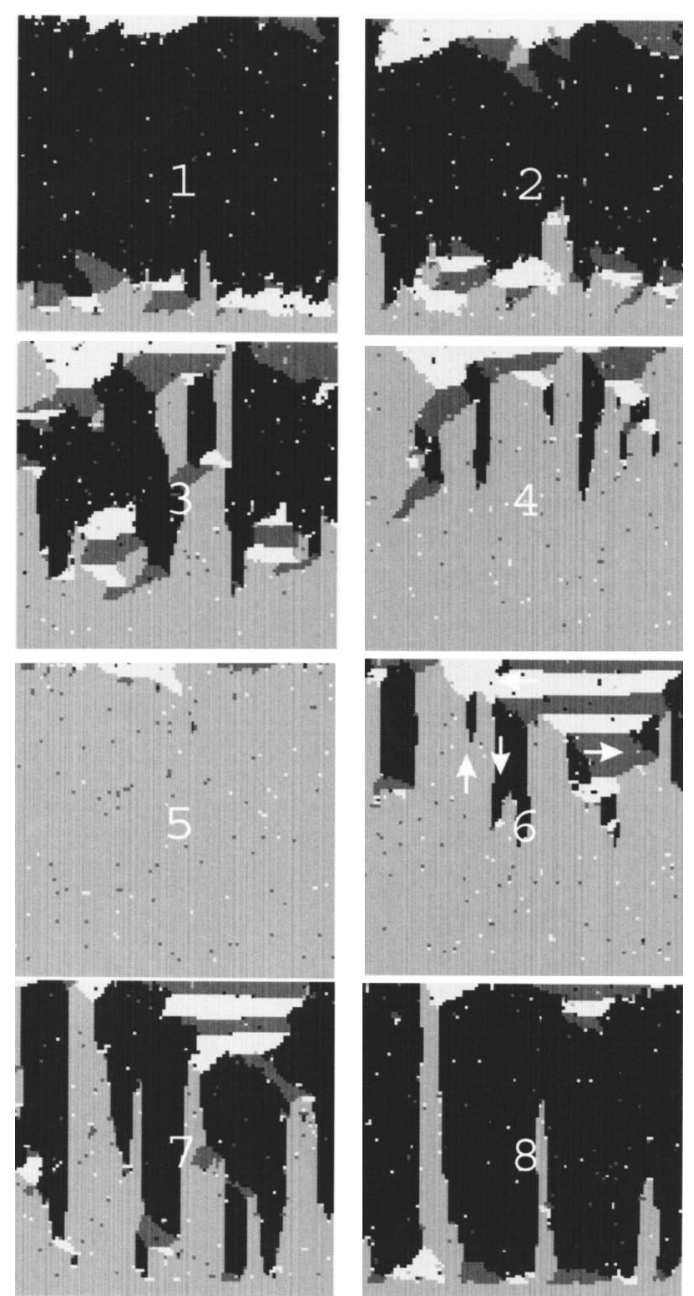

FIG. 3. Domain configurations during switching of a $100 \times 100$ lattice with a frozen-in, downwards polarized bottom interface. The numbers correspond to the points in Fig. 1 (open symbols). The polarization direction in the domains is represented by different shades of gray. The correspondence is visualized in subfigure 6 .

higher, this process occurred only at rather high electric field strengths, entailing a high negative coercive field. Once oppositely oriented domains were formed their growth proceeded very fast due to the high field strengths applied at that point (Fig. 2, parts 3 and 4). The opposite switching process, however, could proceed by heterogeneous nucleation of upwards polarized domains at both interfaces (subfigures 5-8 of Fig. 2); consequently, the positive coercive field is much smaller. Due to the lower electric fields available at that time, the transformation speed is slower, giving rise to a broader transition to positive saturation.

Although the existence of frozen-in interfaces at both the bottom and top electrode is rather unlikely to be realized in actuality, the simulations show the influence of nucleation and growth kinetics on the overall shape of the hysteresis and hint at the possibility that distorted or shifted hysteresis loops might not be the result of internal fields alone, as is generally assumed.

A more realistic example is analyzed in the following. In this case, only one interface at the bottom electrode is assumed to exist. It was assumed that the interface at the bottom electrode is uniformly polarized downwards. This situation might occur at the interface between a thin ferroelectric AIP license or copyright, see http://apl.aip.org/apl/copyright.jsp 
film and the bottom electrode. Due the clamping effect of the substrate this interface is not free, and switching in this thin layer might be impeded. In contrast, the top electrode region in thin films can relax freely. The resulting simulated hysteresis curve is markedly shifted along the electric field axis (Fig. 1, open symbols). Some sample domain configurations are shown in Fig. 3. The enumeration of the subfigures corresponds to the states in Fig. 1 (open symbols). While switching the material from positive remanence to negative saturation (states 1-4), the nucleation and growth of oppositely oriented domains starts from both electrodes. It proceeds faster, however, from the bottom electrode, since here, no new nuclei have to be created. In contrast, the switching process from negative remanence to positive saturation (states 5-8) is somewhat impeded, since the nucleation and growth of new upwards polarized domains proceeds only from the top electrode. No new domains are formed in the frozen-in, downwards polarized bottom interface region. The apparent shift along the field axis of the hysteresis curve is therefore only due to a difference in growth kinetics. It might be argued that the inclusion of a thin, uniformly polarized interface region in itself gives rise to an internal field; however, the evolution of the domain configuration clearly shows that the shift is entirely due to the detailed features of the nucleation and growth process itself. Additionally, the internal field would be mediated by the long-range dipole-dipole interaction, which has been limited to the sixth nearest neighbor in the simulation to save computing time. For a discussion of the legitimacy of this approach, the reader is referred to Ref. 8.

In conclusion, evidence was provided that the nucleation and growth dynamics alone can cause an imprint-like effect in ferroelectrics. This might offer an explanation for the frequently observed phenomenon of initial imprint; that is, an asymmetry of the hysteresis curve in as-prepared ferroelectric thin films. In these systems, a layer of frozen-in polarization close to the bottom electrode might exist due to the clamping effect of the substrate, leading to a switchingdirection-dependent nucleation and growth, entailing a shifted hysteresis loop. Upon further cycling, this layer might relax, thus leading to a symmetrical hysteresis loop.

\footnotetext{
${ }^{1}$ A. Tagantsev, Int. Ferroelectrics 16, 237 (1997).

${ }^{2}$ K. Abe and S. Komatsu, Jpn. J. Appl. Phys. 32, 4186 (1993).

${ }^{3}$ C. Zhou and D. Newns, J. Appl. Phys. 82, 3081 (1997).

${ }^{4}$ A. Tagantsev and I. Stolichnov, Appl. Phys. Lett. 74, 1326 (1999).

${ }^{5}$ M. Grossmann, O. Lohse, D. Bolten, U. Boettger, and R. Waser, J. Appl. Phys. 92, 2680 (2002).

${ }^{6}$ M. Grossmann, O. Lohse, D. Bolten, U. Boettger, and R. Waser, J. Appl. Phys. 92, 2688 (2002).

${ }^{7}$ S. Desu, J. Electrochem. Soc. 140, 2981 (1993).

${ }^{8}$ B. Potter, V. Tikare, and B. Tuttle, J. Appl. Phys. 87, 4415 (2000).

${ }^{9}$ D. Bolten, U. Böttger, and R. Waser, Jpn. J. Appl. Phys. 41, 7202 (2002).
} 